\title{
A Survey on Energy Efficient Protocols for Wireless Sensor Networks
}

\author{
B.Baranidharan \\ Assistant Professor, School of computing, SASTRA \\ University, Tamil Nadu, India
}

\author{
B.Shanthi \\ Professor, School of computing, SASTRA \\ University, Tamil Nadu, India
}

\begin{abstract}
Wireless sensor networks are a web of sensor nodes with a set of processor and limited memory unit embedded in it. Reliable routing of packets from the sensor node to its base station is the most important task for the networks. In wireless sensor networks, routing is bit more complex than other wired or wireless networks. The routing protocols applied for the other networks cannot be used here due to its battery powered nodes. Unlike other wireless networks routing in WSN should be the energy efficient one. This paper gives an overview of the different routing strategies used in wireless sensor networks and gives a brief working model of energy efficient routing protocols in WSN. We have also compared these different routing protocols based on metrics such as mobility support, stability, overlapping. The study concludes with the recommendations to the future direction in the energy efficiency model for the sensor networks.
\end{abstract}

\section{Keywords:}

Wireless sensor networks, mobility, energy efficiency

\section{INTRODUCTION}

The basic idea of anytime and anywhere computing leads to the new field called mobile computing. The advances in the wireless technology are also one of the major stimuli for the growth of mobile computing. But here in this ubiquitous computing environment we can't follow the normal architecture and protocols which have been used in the fixed network due to it's battery powered devices involved in the computing and transmission of the data. The advancement in these miniature computing model and wireless transmission techniques lead to the development of the wireless sensor networks. Sensor networks are needed in the applications like environment monitoring, industrial control units, military applications and in the context aware computing environments.

Since the entire sensor nodes are battery powered devices, energy consumption of nodes during transmission or reception of packets affects the life-time of the entire network. To make routing, an energy efficient one, number of protocols like LEACH and PEGASIS were developed. Though they have achieved efficiency by more than 8 times than the previous protocols, still these are used for only static sensor nodes. In this paper we have proposed a novel approach to develop an energy efficient routing for the mobile sensor networks. The section II in this paper describes the existing routing strategies in WSN and section III gives an overview about energy efficient routing protocols like LEACH, HEED, DECA and PEGASIS. The section IV gives a brief idea about our proposed hybrid model and last section concludes our paper with the future direction in WSN.

\section{ROUTING STRATEGIES IN WSN}

A number of routing protocols have been developed for the WSN till today. Due to its constraints in the processing power and limited battery power, the routing protocols for the wired networks cannot be used here. All the proposed protocols will fall under any of the three categories: 1) Direct approach 2) Location based routing 3) Attribute based routing.

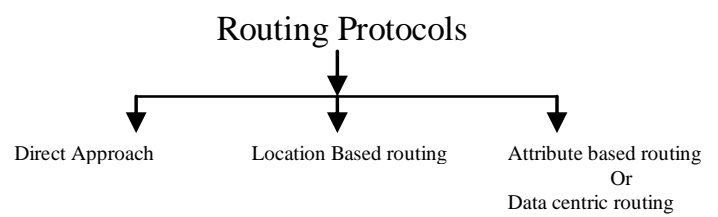

Fig 1-Hierarchical classification of routing strategies

The simple flooding type routing protocols will be coming under the direct approach. Though it is simple in its implementation, it is not an energy efficient protocol for the sensor networks.

In the Location based routing the base station communicates with sensor nodes based on its location identity. Here all the nodes are aware of its location through GPS (Global Positioning System) receivers in the network.

In WSN instead of collecting information from all the nodes the application needs the data only from the nodes which satisfies its interest and this information gathering technique is widely called as the data centric approach or attribute based routing. Direct diffusion and rumor routing are the best examples for the attribute based routing or data centric approach.

\subsection{Location based routing:}

The routing of data to the nodes is done by the geographic location of the nodes (i.e.) nodes are identified by its location only. The location information of the individual nodes is obtained by the low power GPS receivers embedded in the nodes. Some of the most important protocols coming under the 'Location based routing' strategy are 
- Greedy approach

- Compass routing

- DREAM

- GPSR

- GEAR

In the above mentioned protocols the first two follows the single path approach and next three follows the multi-path or flooding mechanism.

2.1.1 Greedy approach: In [17] I. Stojmenovic et al. stated that the neighboring node $\mathrm{Y}$ which is closer to the destination node $\mathrm{D}$ from the source or intermediate node $\mathrm{S}$ conducts the packet to the destination. The data flows through the intermediate nodes like this until it reaches the destination node D.

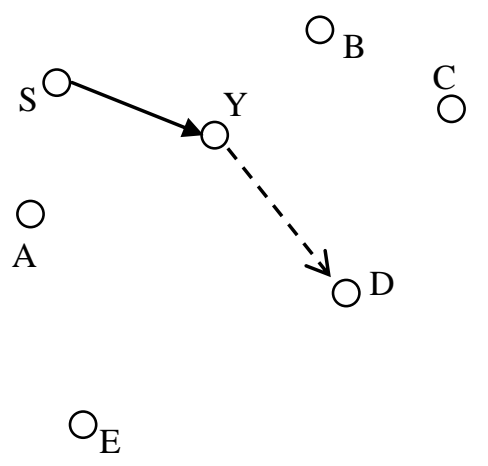

Fig 2 - Implementation of Greedy approach in WSN

2.1.2 Compass routing: In [16] E.Kranakis et.al stated that the source node $\mathrm{S}$ calculates the direction of the destination $\mathrm{D}$ and the neighboring node $\mathrm{Y}$ which is having closest direction to the destination than SD is selected as the next eligible intermediate node to route the data from the source node.

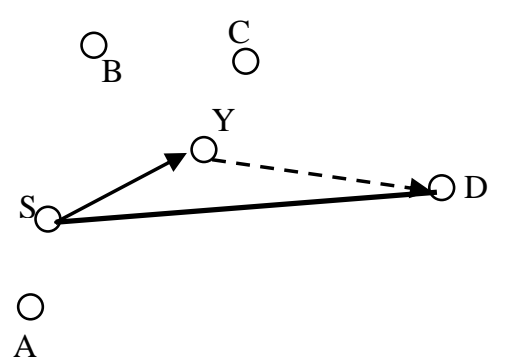

Fig 3 - Compass routing approach in WSN

2.1.3 DREAM [A distance Routing Effect Algorithm for Mobility]: In [18] S.Basangi proposed the model of flooding packets to all the neighboring nodes of node $\mathrm{x}$, here a different approach was taken. The data is only flooded to the limited number of nodes which is coming under the area when the tangents are flowing between the node $\mathrm{x}$ to the circle centered at destination D. Since we are forwarding data to limited number of nodes, it is a better one than its predecessors.

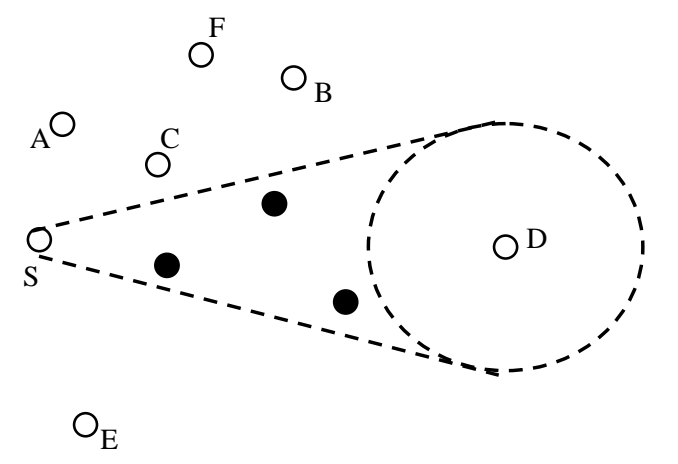

Fig. 4 - Routing structure in DREAM protocol

Here in the above diagram the dotted nodes which fall under the tangents connecting the circle and the source node is only receiving the data.

2.1.4 GPSR [Greedy Perimeter Stateless Routing]: The modified version of greedy-face-greedy algorithm is the Greedy perimeter stateless routing[14]. Here the combination of greedy and perimeter approach is taken. Initially the data is forwarded by using greedy approach and if the packet gets stuck at any point, perimeter approach comes to rescue of the situation. But this perimeter approach is followed till a node closer to the destination was found than the node at which the packet got stuck. It ensures the guaranteed delivery of packets to the destination.

\subsubsection{GEAR [Geographic \& Energy Aware} Routing]: In [11] Y.Yu et. al takes the different approach than its previous protocols by considering the least cost path to route the packets to the destination node which is identified by its location information.

2.1.6 GAF[Geographic Adaptive Fidelity]: In [12] $\mathrm{Y} . \mathrm{Xu}$, D.Estrin et. al proposed that the nodes coming under the particular geographical range will be associated with a particular grid. The communication cost of nodes coming under the same grid will be same. During the routing decision any one node from the particular grid will wake up and takes part in routing and all other nodes in the same grid will go to the sleeping state to avoid unnecessary energy depletion.

\subsection{Data centric routing}

The basic view which separates the sensor network from the other wireless networks is the distinction between the address of the node and the content of the node. Here in the above said 'Location based routing' strategy the entire sensor nodes in the particular region are viewed as the system rather than as the separate nodes. This lead the sensor network to take routing decisions based on data held by the nodes in the network rather than its destination address or geographic location. Some of the protocols which follow the data centric routing are, 
- $\quad$ Directed diffusion

- SPIN

- $\quad$ Rumor routing

2.2.1 Directed Diffusion: In direct diffusion [13] the data generated in the nodes is identified by its attribute-value pair. Here the base station passes its 'interest' all through the network. The issued user 'interest' would be traveling all through the sensor networks and compared with the event record in the concerned node. If the event record matches with the 'interest' the event record is sent to the base station otherwise the 'interest' is passed to the neighboring nodes. Here the use of gradients is an important factor in the direct diffusion technique. When the source node is responding to the base station, it will be receiving the data from multiple routes and again the base station have to select the gradient which is having minimum delay time than others. The elements used in Directed Diffusion are,

Interest propagation: The task is represented by the attributevalue pair and diffused through the network.

Data propagation: When the user 'interest' matches with the event record, the data are forwarded back to the base station.

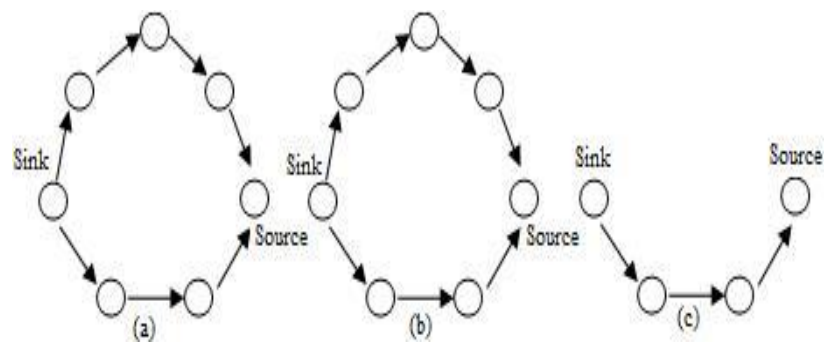

Fig-5 Directed Diffusion- (a) Interest Propagation, (b) gradient formation and (c) selection of optimum return path.

2.2.2 SPIN : 'Sensor Protocols for Information Negotiation' [8] is the family of protocols based on data centric approach. It is also called as the 3 -stage protocol since 3 subsequent steps are involved in data transformation between the nodes. When the node generates information, it is intimated to its 1-hop neighbors using ADV (advertisement) packet and if the neighbor node is in need of the information it will request the data through REQ (request) packet. Finally the original DATA packet will be sent to the neighbor node. Using this protocol redundancy in information is avoided in the sensor networks. The SPIN node will only take the data from its 1-hop neighbor nodes and only forward the best available data to the base station. The main drawback in this method is if a node which is in need of the data can't receive the data when it is not the 1-hop neighbor node to the source node which generates the required data.

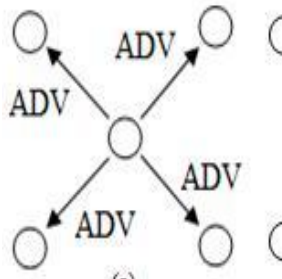

(a)

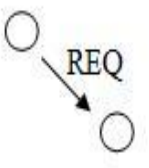

(b)

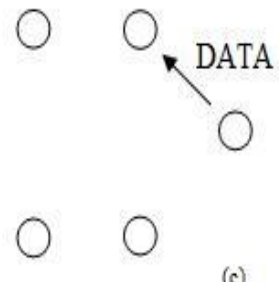

(c)
Fig 6-SPIN - (a) Data advertisement (ADV), (b) request (REQ) and (c) transferring of original DATA

2.2.3 Rumor routing: As a further step apart from base station as the only initiator in information gathering for the data, the source node also acts as the initiator in passing their sensed data to the base station and this technique is widely called as the rumor routing[10]. Here, in this approach the data collected by the sensor nodes will be sent to its neighboring nodes and it goes on till reaches the interested region or the end node of the network. At the same time the user interest is also sent through the network. When the two regions meet each other required data are gathered and given to the base station.

\section{ENERGY EFFICIENCY IN ROUTING}

Since energy efficiency is more important for wireless sensor networks than any other networks, more research works have already been done in routing in WSN. In general, data transmission in wireless communication takes more power than data processing. Whenever the nodes are transmitting more number of data proportionately their battery power also get reduced. To reduce the data size we can go for data fusion or aggregration techniques. Data fusion is that in which the sensed data from different nodes are fused at certain point suitable for the transmission in its reduced size.

Even in the data aggregration concept there are two types of aggregration. The first type of data aggrgration fuses the data gathered from different sources and sends the final fused data in reduced size. But the problem behind this approach is it lacks in accuracy and precision of data from various sensor nodes. The second approach combines the data from different sources under the the single header and forward it to the base station. Here header packets consolidates and pass it to the base station without any modification to the original data from the sensors. Hence accuracy is improved.

Study on energy efficient routing in WSN brings this two broad classification of approaches. They are,

- Clustering approach

- Tree based approach

\subsection{Clustering techniques:}

Dividing the sensor networks into small manageble units is called as clustering. Though the main reason behind the implementation of the clustering scheme is to improve the scalability of the network, it is an important factor in achieving energy efficient routing of data within the network. Apart from achieving scalability of the network it has more advantages like conserving communication bandwidth within the clusters, 
avoiding redundant message transfer between the sensor nodes, localizing energy efficient route setup within the clusters. Some of the energy efficient routing protocols based on clustering are LEACH, HEED, DECA, etc...

\subsubsection{LEACH:}

Low energy adaptive clustering hierarchy[9] uses the clustering principle to distribute the energy consumption all along its network. Here, based on data collection, network is divided into Clusters and Cluster heads are elected randomly. The cluster head collects the information from the nodes which are coming under its cluster. Let us see the steps involved in each round in the LEACH protocol.

Advertisement phase: This is the first step in LEACH protocol. The eligible cluster head nodes will be issuing a notification to the nodes coming under its range to become a cluster member in its cluster. The nodes will be accepting the offer based upon the Received Signal Strength (RSS).

Cluster set-up phase: In this step the nodes will be responding to their selected cluster heads.

Schedule creation: After receiving response from the nodes the cluster head have to make a TDMA scheme and send back to its cluster members to intimate them when they have to pass their information to it.

Data transmission: The data collected by the individual sensors will be given to the cluster head during its time interval and on all other time the cluster members radio will be off to reduce it energy consumption.

Here in the LEACH protocol multi cluster interference problem was solved by using unique CDMA codes for each cluster.

It helps to prevent energy drain for the same sensor nodes which has been elected as the cluster leader, using randomization for each time cluster head would be changed. The cluster head is responsible for collecting data from its cluster members and fuse it. Finally each cluster head will be forwarding the fused data to the base station. When compared with its previous protocols LEACH have shown a considerable improvement.

\subsubsection{HEED:}

Though the LEACH protocol is much more energy efficient when compared with its predecessors the main drawbacks in this approach is the random selection of cluster head. In the worst case the $\mathrm{CH}$ nodes may not be evenly distributed among the nodes and it will have its effect on the data gathering. To avoid the random selection of $\mathrm{CHs}$ a new algorithm called HEED[6] was developed which selects the CHs based on both residual energy level and communication cost. The HEED protocol get executed in three subsequent phases,

Initialization phase: During this phase the initial $\mathrm{CHs}$ nodes percentage will be given to the nodes. It is represented by the variable $\mathrm{C}_{\text {prob }}$. Each sensor node compute its probability to become $\mathrm{CH}$ by the formula, $\mathrm{CH}_{\text {prob }}=\mathrm{C}_{\text {prob }} * \mathrm{E}_{\text {residual }} / \mathrm{E}_{\max }$ where $\mathrm{E}_{\text {residual }}$ to residual energy level of the concerned node, $\mathrm{E}_{\max }$ corresponds to maximum battery energy. Since HEED supports heterogeneous sensor nodes $\mathrm{E}_{\max }$ may vary for different nodes according to its functionality and capacity.

Repetition phase: Until the $\mathrm{CH}$ node was found with the least transmission cost, this phase was iterated. If the node cannot find the appropriate $\mathrm{CH}$, then the concerned node itself was selected as the $\mathrm{CH}$.

Finalization phase: The selection of $\mathrm{CH}$ is finalized here. The tentative $\mathrm{CH}$ now becomes the final $\mathrm{CH}$ node.

\subsubsection{DECA:}

DECA is an improved Distributed Efficient Clustering Approach[5]. The basic difference between the HEED and DECA is how the nodes take the decision and the score computation. The phases involved in DECA operations are,

Start Clustering: In the initial phase all the nodes will compute its score with the help of the function score $=\mathrm{w}_{1} \mathrm{E}+\mathrm{w}_{2} \mathrm{C}+\mathrm{w}_{3} \mathrm{I}$. E corresponds to residual energy, $\mathrm{C}$ stands for node connectivity, and I stand for node identifier. After some delay time the score value will be given to the neighboring nodes with the node ID and cluster ID if the computed score is a higher value.

Receive Clustering Message: When the node is receiving the score value higher than it and if it is not attached to any cluster it accepts the sender node as its $\mathrm{CH}$.

Actual announcement: After the completion of second phase, when new nodes and already exciting nodes from some other cluster forming a cluster with a new head, the CHs ID, cluster ID and score value should be broadcasted.

Finalize Clustering: This is the same as HEED protocol that the new cluster with its head is finalized for all other nodes.

\subsection{Tree Based Approach:}

Apart from clustering techniques in WSN, another energy efficient way of routing the data over the network is tree based approach. In this approach a hierarchical manner of aggregation points are formed which resembles the tree structure. The leaves are the source nodes and the root is the sink node. The data when travelling gets aggregated in the intermediate nodes itself. The most successful energy efficient routing protocol which follows the tree based approach was PEGASIS.

\subsubsection{PEGASIS:}

Though the Cluster based protocols like LEACH have shown a factor of 8 improvements when compared with its previous protocols further improvements were done by forwarding the packets to only one neighbor of the node. This method had been named as 'Power Efficient Gathering in Sensor Information System' [7]. Instead of forwarding the packets from many cluster heads as like in LEACH protocol here in PEGASIS each node will form a chain structure to the base station through which the data would be forwarded to the BS node.

Here in PEGASIS energy efficient is achieved by transmitting the data to only one of its neighbor node. There the collected 
data is fused and the fused data will be forwarded to its immediate one hop neighbor. Since all the nodes are doing the data fusion at its place there is no rapid depletion of power for the nodes present near the Base station. Also in this method each node will be getting the chance to forward the gathered data to the base station.

But when the sensor measurements are aggregated to be a single packet, only fraction of the data generated by the sensor is given to the base station. In some applications when the particular sensor measurement is needed it fails to give it to base station. But apart from the function of the routing protocol we can make the sensor network database to follow the multi resolution scheme where the aggregated data will be present in the root node and the finer data can be obtained by further tree traversal mechanism.

Though the Directed Diffusion[13] and Rumor routing[10] techniques comes under tree based approach in terms of energy efficiency it lacks behinds PEGASIS model.

Table 1 Comparison of the protocols in terms of its related parameters

\begin{tabular}{|l|l|l|l|l|}
\hline $\begin{array}{l}\text { PROTOCO } \\
\text { L }\end{array}$ & $\begin{array}{l}\text { Latency in } \\
\text { the sensor } \\
\text { network }\end{array}$ & $\begin{array}{l}\text { Mobilit } \\
\text { support }\end{array}$ & $\begin{array}{l}\text { Cluster } \\
\text { Stability }\end{array}$ & $\begin{array}{l}\text { Distribute } \\
\text { d cluster } \\
\text { heads }\end{array}$ \\
\hline $\begin{array}{l}\text { Direct } \\
\text { Approach }\end{array}$ & Low & Nil & N/A & N/A \\
\hline $\begin{array}{l}\text { Directed } \\
\text { Diffusion }\end{array}$ & Higher & Nil & N/A & N/A \\
\hline $\begin{array}{l}\text { Rumor } \\
\text { routing }\end{array}$ & $\begin{array}{l}\text { Acceptabl } \\
\text { e }\end{array}$ & Nil & N/A & N/A \\
\hline $\begin{array}{l}\text { LEACH } \\
\text { e }\end{array}$ & $\begin{array}{l}\text { Acceptabl } \\
\text { e }\end{array}$ & Nil & $\begin{array}{l}\text { Moderat } \\
\text { e }\end{array}$ & Moderate \\
\hline HEED & $\begin{array}{l}\text { Acceptabl } \\
\text { e }\end{array}$ & Nil & Good & Good \\
\hline DECA & Higher & Nil & N/A & N/A \\
\hline PEGASIS & & & \multicolumn{2}{|l}{} \\
\hline
\end{tabular}

\section{PROPOSED MODEL}

The existing energy efficiency model for the sensor network shows considerable improvement in one or more objectives to suite the specific application, still there needs a lot of work to be done on energy efficient model in terms of low clustering overhead, distributed cluster heads, continuous packet delivery, reduced data fusion cost. In this paper we are proposing a new hybrid protocol model which considers all these factors in the routing mechanism for the wireless sensor network. The following are the steps involved for the proposed hybrid model.

1. Clustering based on k-means algorithm.

2. Improved cluster head selection through RSS (Received Signal Strength) value.

3. Alternate $\mathrm{CH}$ (Cluster Head) selection for continuous packet delivery.
4. Shortest path to the super cluster further reduces the power consumption.

5. Compression techniques for reduced data fusion cost.

By incorporating small changes in each step, we hope this hybrid model may improve the efficiency of routing protocol for Wireless sensor networks.

\section{CONCLUSION}

Thus the existing routing strategies in the wireless sensor networks and their corresponding protocols had been explained. Though the protocol like LEACH, HEED, DECA, SPIN, and PEGASIS are proved to be energy efficient than its previous models the main pitfalls in these protocols are that nodes are assumed to be static and stationary. The energy efficiency model is untested while the sensor nodes exhibit mobility. Future works may concentrate on achieving better energy efficiency in routing mechanism for mobile wireless sensor nodes.

\section{REFERENCES}

[1]. Yi-hua zhu, Wan-deng wu, Jian pan, Yi-ping tang, "An energy efficient data gathering algorithm to prolong lifetime of wireless sensor networks", Computer Communications 33(2010) 639-647.

[2]. F.Bajaber, I.awan, "Centralized dynamic clustering for wireless sensor network" in International Conference on Advanced Information Networking and Applications Workshops, 2009, pp 193-198.

[3]. Ameer Ahmed Abbasi, Mohamed Younis, "A Survey on Clustering Algorithms for Wireless Sensor Networks", Computer Communications 30 (2007) 2826-2841.

[4]. Kemal Akkaya, Mohamed Younis, "A Survey on routing protocols for Wireless Sensor Networks", Ad Hoc Networks 3 (2005) 325-349.

[5]. Miau Yu, Jason H.Li and renato Levy, "Mobility Resistant Clustering in Multi-Hop Wireless Networks", Journal of Networks, Vol.1, No.1, May 2006.

[6]. O. Younis, S. Fahmy, "HEED: A Hybrid,EnergyEfficient, Distributed clustering approach for Ad Hoc sensor networks", IEEE Transactions on Mobile Computing 3 (4) (2004) 366-379.

[7]. S. Lindsey, C. Raghavendra,"PEGASIS: PowerEfficient Gathering in Sensor Information Systems," IEEE Aerospace Conference Proceedings, 2002, Vol. 3. No. 9-16, pp. 11251130.

[8]. J. Kulik, W. R. Heinzelman, and H. Balakrishnan, "Negotiation-based protocols for disseminating information in wireless sensor networks," Wireless Networks, Volume: 8, pp. 169-185, 2002. 
[9]. W.B. Heinzelman, A.P. Chandrakasan, H.Balakrishnan , "Application specific protocol architecture for wireless microsensornetworks", IEEE Transactions on Wireless Networking (2002).

[10].D. Braginsky, D. Estrin," Rumor Routing Algorithm for Sensor Networks," Proceedings of the 1 st Workshop on Sensor Networks and Applications (WSNA'02), Atlanta, GA, Oct.2002.

[11].Y. Yu, D. Estrin, and R. Govindan," Geographical and Energy-Aware Routing: A Recursive Data Dissemination Protocol for Wireless Sensor Networks", UCLA Computer Science Department Technical Report, UCLA-CSD TR-01-0023, May 2001.

[12].Y. Xu, J. Heidemann, D. Estrin,” Geographyinformed Energy Conservation for Ad-hoc Routing," In Proceedings of the Seventh Annual ACM/IEEE International Conference on Mobile Computing and Networking 2001, pp. 70-84.

[13].Intanagonwiwat, C. Govindan R. and Estrin,D. "Directed Diffusion: A Scalable and Robust Communication Paradigm for Sensor Networks". In Proceedings of the Sixth Annual International Conference on Mobile Computing and Networks (MobiCOM 2000), August 2000, Boston, Massachusetts.
[14].B. Karp and H. T. Kung, "GPSR: Greedy perimeter stateless routing for wireless sensor networks", in the Proceedings of the 6th Annual ACM/IEEE International Conference on Mobile Computing and Networking (MobiCom '00), Boston, MA, August 2000.

[15].P.Bose and P.morin. "Online routing in triangulation". In Proc. $10^{\text {th }}$ International Symbosium on Algorithm and Computation(ISAAC '99), pages 113-122. SpringerVerlag, LNCS 1741, 1999.

[16].E.Kranakis, H.Singh and J.Urrutis. "Compass routing on geometric networks. In proc. $11^{\text {th }}$ Canadian conference on Computational Geometry", Pages 5154, Vancouver, August 1999.

[17].I. Stojmenovic and X. Lin. "GEDIR: Loop-Free Location Based Routing in Wireless Networks", In International Conference on Parallel and Distributed Computing and Systems, Boston, MA, USA, Nov. 36, 1999.

[18].S. Basagni and et. al. A Distance Routing Effect Algorithm for Mobility (DREAM). In ACM/IEEE Int. Conf. on Mobile Computing and Networking ( MobiCom'98), October 1998 